\title{
Percepciones de Futuros Maestros ante Nuevos Contextos de Aprendizaje
}

\author{
Myriam Alvariñas y María A. Fernández-Villarino \\ Facultad de Ciencias de la Educación y del Deporte, Universidad de Vigo, Campus Universitario \\ A Xunqueira s/n, 36005 Pontevedra-España (e-mail: myalva@uvigo.es)
}

Recibido Mar. 10, 2011; Aceptado Abr. 13, 2011; Versión final recibida May. 07, 2011

\begin{abstract}
Resumen
Este trabajo presenta y analiza la opinión de 94 estudiantes de la titulación de maestro en Educación Infantil y de la titulación de maestro en Educación Primaria, sobre el uso de sesiones no presenciales y la repercusión de estas estrategias en su formación. Utilizando como instrumento un cuestionario, se obtiene información sobre dichas clases no-presenciales. Las actividades más requeridas a los estudiantes se vinculan al trabajo online, al análisis de documentos y a la realización de trabajos individuales. Las competencias más valoradas son el aprendizaje autónomo, la búsqueda de información y el uso de nuevas tecnologías. Los estudiantes establecen una relación positiva entre el uso de estas estrategias y la mejora de su proceso de enseñanza-aprendizaje. Por esta razón, varios profesores se están pensando implantar este tipo de metodologías activas en sus cursos.
\end{abstract}

Palabras clave: sesiones no presenciales, enseñanza-aprendizaje, nuevas tecnologías, Internet

\section{Future Teacher's Perceptions in New Learning Contexts}

\begin{abstract}
This paper presents and analyzes the opinion of 94 pre-school and primary education internship teachers about the use of non-face sessions and the influence of these strategies on their formation. Using a questionnaire as the main instrument, information about the non-face classes is obtained. The activities that the students developed more frequently are related to on-line work, analysis of documents and individual reports. The most valued competences are the autonomous work, the searching of information and the use of new technologies. The students establish a positive relationship between the use of these strategies and the improvement of their teachinglearning process. For this reason, several professors are thinking of using theses active methodologies in their courses.
\end{abstract}

Keywords: non-face sessions, teaching-learning, new tecnologies, Internet 


\section{INTRODUCCIÓN}

Los tiempos que corren están exigiendo de quienes se dedican a la formación de profesionales un estado de permanente alerta, de reconceptualización e indagación de nuevas y viejas herramientas, a la luz de las demandas sociales de aprender "de otra manera", aquella que tenga en cuenta las exigencias de los nuevos contextos de enseñanza y aprendizaje caracterizados por la integración de las nuevas tecnologías y, sobre todo, por el ineludible protagonismo de sus participantes. (Agra et al., 2003). El proceso de adaptación al Espacio Europeo de Educación Superior (EEES) en el que se encuentran inmersas las universidades españolas, y concretamente la Universidad de Vigo, ha llevado al profesorado a desarrollar diferentes acciones de innovación educativa que mejoren los procesos formativos que se desarrollan. Motiva la mejora de la calidad docente, lo que hace que se investigue sobre la propia acción y que se dé un paso más hacia las necesidades metodológicas de este nuevo espacio educativo.

Una de las iniciativas que se han desarrollado ha sido la de utilizar nuevos recursos didácticos que provoquen el aprendizaje y que hagan del alumnado el protagonista de su desarrollo formativo. Por medio de estos recursos, se pretende contribuir a la adquisición de competencias en las que el aprendizaje autónomo y la responsabilidad del proceso sean ejes vertebradores de la educación. La intencionalidad metodológica de esta experiencia, tiene que ver con los trazos definidos por Fernández March (2006) en donde el nuevo modelo educativo es más eficaz si, entre otros, se desarrollan modelos centrados en el aprendizaje del alumnado, si el trabajo es cooperativo y si se da protagonismo a las tecnologías de la información y de la comunicación.

Para responder a esta nueva forma de pensar y trabajar, optando por incorporarse poco a poco a un nuevo paradigma, se han puesto en marcha en nuestras aulas las sesiones no presenciales. Estos sistemas de enseñanza, surgen con el objetivo de que la educación llegara a todas las personas (Sangrà, 2002) y como señala Santángelo (2000) fueron considerados en su día como de nivel o calidad menor a la enseñanza presencial. Sin embargo, en la actualidad, y auspiciados por el marco Europeo de Educación Superior, a los docentes se les insta a poner en marcha este tipo de sesiones, con el fin de contribuir a la creación de un sistema más abierto, autónomo y colaborativo, de manera que sean un complemento, y nunca una sustitución, de la modalidad presencial; así lo refleja Bolívar (2009) cuando dice que dentro del proceso abierto de la Declaración de Bolonia (DB, 1999) la actividad docente adquiere nuevos enfoques, entre ellos, la coordinación entre actividad presencial y no presencial. Y este hecho está íntimamente relacionado con lo que significa el ECTS, Sistema Europeo de Transferencia de Créditos, (R.D. 1125/2003), un sistema centrado en el estudiante, que se basa en la carga de trabajo (tiempo invertido tanto en actividades presenciales como no presenciales) necesario para que se consigan los objetivos de un programa de estudios; cada crédito son 25-30 horas de trabajo del estudiante.

Las actividades presenciales son aquéllas que reclaman la intervención directa de profesores y estudiantes como son las clases teóricas, los seminarios, las clases prácticas, las prácticas externas y las tutorías. Se consideran como modalidades no presenciales las actividades que los estudiantes pueden realizar libremente bien de forma individual o mediante trabajo en grupo. (De Miguel, 2005). Esta forma de diferenciar las clases tiene en cuenta si el docente y el estudiante comparten un espacio y un tiempo determinados o no. Al hablar de no presencialidad uno se puede referir o bien a que ambos agentes no comparten ninguno de los dos elementos (espaciotiempo) o bien a que están separados en distancia pero comparten el mismo tiempo (por ejemplo, tutorando trabajos o resolviendo dudas mediante una plataforma de tele-enseñanza en ciertas horas prefijadas o realizando actividades por videoconferencia). Gallego (2007) indica que el modelo constructivista en los procesos formativos universitarios se basa en procesos dinámicos en los que la información se interpreta y reinterpreta de manera que el estudiante va construyendo su propio conocimiento. Este modelo es el que se promueve ya desde hace años mediante la influencia del EEES y en él, el material y los recursos se constituyen como canales de primer orden, sobre todo en la enseñanza a distancia; así en muchas de sus declaraciones y comunicados como el de Lovaina (2009), se promulga esta necesidad de nuevos enfoques de enseñanza-aprendizaje. 
Mediante el uso de las sesiones no presenciales se contribuye a la consecución de algunas de las competencias que se le exigen al "nuevo docente" relativas al uso de las nuevas tecnologías, la revalorización de la función tutorial o la elaboración de materiales que faciliten el aprendizaje autónomo y colaborativo. Se convierten en tutores, orientadores, coordinadores y asesores (Zabalza, 2003). En definitiva, una nueva forma de entender el rol que se desarrolla en la acción educativa, un papel más intencional y comprometido que permita alcanzar el éxito en esta labor.

Fernández March (2008) propone diversos métodos activos y sus características, así como De Miguel (2005) plantea modalidades más adecuadas para las actividades presenciales y otras para el trabajo autónomo (estudio y trabajo en pequeño grupo, como preparación de trabajos, seminarios, investigaciones o lecturas; y estudio y trabajo autónomo como estudio personal, realización de ejercicios o trabajo en biblioteca). Alados et al. (2010) destacan entre otras actividades propias de las sesiones no presenciales, la lectura de bibliografía, la resolución de problemas, la realización de informes escritos o la búsqueda bibliográfica. Asimismo, Del Río (2010) utiliza las horas no presenciales para la búsqueda y recopilación de material, el planteamiento de ideas a tratar a posteriori con el grupo-clase o para completar los trabajos iniciados y continuados en clase.

Las últimas investigaciones realizadas en este campo muestran que cada vez se utiliza más este tipo de recurso en el sistema universitario y que la no presencialidad de las sesiones está asociada de manera significativa a entornos virtuales de enseñanza-aprendizaje. Así, algunos trabajos indagan acerca del desarrollo de competencias en grupos de trabajo presenciales y no presenciales, trabajando estos últimos a través de una plataforma virtual (García et al., 2008); otros, valoran la utilización del portafolio como herramienta para analizar la formación on-line y presencial (Agra et al., 2003); o investigan sobre la aplicación de materiales en la red (Castañeda, 2007); también existe interés por conocer los entresijos del trabajo colaborativo en este tipo de modalidad formativa (Alfageme, 2005). En esta línea, se puede observar que algunos de estos estudios se centran en la percepción de los estudiantes (Castañeda, 2007; Chiecher et al., 2005; Lamas et al., 2010; Pérez-Lorido, 2008), del profesorado (Gallego, 2007; Pérez-Lorido, 2007), o bien revelan el pensamiento de ambos agentes (Barceló et al., 2007; Blasco y Mengual, 2007; Fernández-Villarino, 2008; Mondéjar et al., 2007) a la hora de afrontar estas experiencias de carácter no presencial.

Por norma general, las experiencias son satisfactorias para las personas protagonistas del proceso (Alados et al., 2010; Blasco y Mengual, 2007; Chiecher et al., 2005; Fernández-Villarino, 2008; Gras-Martí et al., 2004; Pérez-Lorido, 2008) y el profesorado las valora de manera positiva, haciendo hincapié en su utilidad como complemento de las actividades presenciales, el fomento del aprendizaje colaborativo (Mondéjar et al., 2007), el enriquecimiento de las relaciones docentediscente, el planteamiento de nuevas posibilidades didácticas, las virtudes derivadas de la desubicación geográfica del conocimiento, etc. (Pérez-Lorido, 2007). Sin embargo, este tipo de trabajo, que en muchas ocasiones se corresponde con experiencias de índole exploratoria, no está exento de dificultades. De este modo, se critica el amplio volumen de trabajo, los problemas de acceso y disponibilidad de recursos, (Mondéjar et al., 2007), la poca experiencia del alumnado para emplear los nuevos medios digitales, la débil comunicación en línea entre profesores/as y alumnos/as a través de herramientas como los foros o los debates (Castañeda, 2007) o la dedicación excesiva que requiere una buena atención de las tutorías virtuales (Pérez-Lorido, 2007).

Respecto a la contribución de los recursos virtuales a la mejora de las relaciones entre compañeras/os y profesoras/es, en casos como los de García et al. (2008) se detectan problemas de coordinación y de unificación de criterios; también, en lo que atañe al trabajo colaborativo no presencial (Alfageme, 2005) se aprecia la escasa labor llevada a cabo por los grupos, la participación desigual de los componentes de los mismos, los retrasos derivados de la lentitud al realizar las tareas o las interpretaciones erróneas que se dan en este tipo de comunicación. Por eso, y entre otros motivos, los autores y autoras coinciden en afirmar que la no presencialidad debe coexistir con los sistemas presenciales; aunque, tal y como indica Pérez-Lorido (2007), hemos de tener en cuenta que ese cambio hacia los requerimientos formativos de las personas 
que viven en la sociedad informacional deben de ponerse en marcha con cautela, elaborando propuestas didácticas racionales y equilibradas que vayan más allá de la incorporación vacía de nuevos recursos.

Es importante mencionar también, la dificultad que todavía existe de desprenderse de los modelos tradicionales en los que el docente es el transmisor y controlador del conocimiento (FernándezVillarino, 2008) y es que las rutinas académicas adquiridas a lo largo de los años no favorecen la migración hacia modelos alternativos de enseñanza-aprendizaje (Pérez-Lorido, 2008). No obstante, en trabajos como el elaborado por Blasco y Mengual (2007, p.11) se vislumbra “...que podríamos estar asistiendo a una trasformación del pensamiento del alumno en cuanto a su formación, al considerar éste que la adquisición de conocimiento ya no procede únicamente del profesor y de los libros, sino que él mismo puede contribuir en la generación de su propio conocimiento"

\section{METODOLOGÍA}

Se realiza un análisis descriptivo a partir de la información suministrada por una encuesta. Este tipo de metodología es de carácter fácil y directo, lo que hace que sea muy utilizada en el ámbito educativo. Respondiendo a los objetivos planteados por Cohen y Manion (1990) de describir, comparar, clasificar, analizar e interpretar, su uso nos permitió describir la naturaleza de las condiciones de aplicación de la estrategia didáctica, identificar los valores estándar con los que poder comparar las condiciones existentes y determinar las relaciones entre los elementos estudiados.

Instrumento de recogida de datos:

Con la intención de obtener información detallada y precisa por parte de los estudiantes de cómo se desarrollaron las sesiones y de conocer la repercusión que éstas tenían en el proceso de enseñanza-aprendizaje, utilizamos un cuestionario como estrategia de recogida de datos. Un instrumento similar ya había sido utilizado y validado en un estudio anterior (Fernández-Villarino, 2008) con la misma intencionalidad: obtener información detallada y precisa de cómo se están desarrollando y de la repercusión que tienen en el proceso de enseñanza-aprendizaje, las sesiones no presenciales.

En esta ocasión, se utilizó un cuestionario compuesto por siete preguntas cerradas; para su validación, se recurre al juicio de expertas y expertos tanto metodológicos como del tema que se trataba, para que emitieran su opinión sobre el mismo y aconsejaran sobre las posibles modificaciones que se debían realizar. Asimismo, para la validación de contenido, se preguntó a dos grupos de estudiantes de Educación Primaria e Infantil (veinte personas de cada titulación) diferentes a los que posteriormente participarían en el estudio acerca de la comprensión del cuestionario. El resultado final, fue el instrumento que se administra a la muestra de esta investigación (se trata de una muestra representativa de la población a estudiar). Las estrategias que se emplearon para el análisis de los datos, tenían que ver con el tipo de preguntas que se estaban analizando. En este caso se introdujeron en una base de datos que se diseña para ello. La primera fase del análisis estadístico, se correspondió con una descripción de la muestra mediante la estadística descriptiva, lo que permitió sintetizar la información revelada. El programa utilizado fue el paquete de análisis estadístico SPSS y en la versión 18.0. Las estrategias que se emplearon en el análisis fueron las frecuencias de aparición de cada una de las variables.

\section{Elementos de estudio y sujetos:}

Los objetivos del estudio hacen referencia a los siguientes elementos: nivel de uso de la estrategia, tipos de actividades realizadas, horarios de realización, desarrollo de competencias, valoración del trabajo docente, influencia en el proceso de enseñanza-aprendizaje y expectativas futuras de uso. Los sujetos encuestados son alumnas y alumnos de tercer curso de las titulaciones de Maestro especialista en Educación Infantil y Primaria de la Facultad de Ciencias de la Educación y del Deporte de la Universidad de Vigo. La muestra invitada se correspondía con la 
población objeto de estudio (108 estudiantes de Primaria y 78 de Infantil; un total de 186 personas), pero teniendo en cuenta la no obligatoriedad de la asistencia a las clases en la Universidad de Vigo, respondieron al cuestionario 94 estudiantes, distribuidos en: 50 personas de la especialidad de Educación Primaria y 44 de Educación Infantil.

\section{RESULTADOS Y DISCUSIÓN}

Cerca del $80 \%$ de las personas encuestadas (94) ha utilizado durante su formación estas sesiones, lo que indica que hay estrategias didácticas que ya se están poniendo en marcha antes de que los estudios de grado hayan sido implantados en su totalidad. Nuestros datos son del curso 08/09 y el grado se implanta en el curso siguiente (09/10). Estos estudios "de grado" se corresponden con la nueva estructuración de la enseñanza superior en el modelo europeo, en concreto con su primer nivel, que permitirá el acceso a una titulación con calificación profesional en el mercado laboral europeo (en cuatro años se obtiene el título universitario oficial de graduado/a). Aunque el desarrollo del uso de las nuevas metodologías se indica para los estudios de grado, dado que son ellos los que forman parte del nuevo mapa de titulaciones del EEES y como tal del nuevo paradigma de formación, la necesidad de tener competencia en el desarrollo de estos recursos, lleva al profesorado a experimentar y evaluar la puesta en marcha y funcionamiento en los modelos formativos actuales.

Respecto al tipo de actividades realizadas en dichas sesiones, los estudiantes encuestados invierten el tiempo principalmente en: buscar información a través de Internet (90\%), leer o analizar documentos (80\%), llevar a cabo trabajos de tipo individual (74\%) y realizar o entregar ejercicios por medio de una plataforma virtual (73\%).

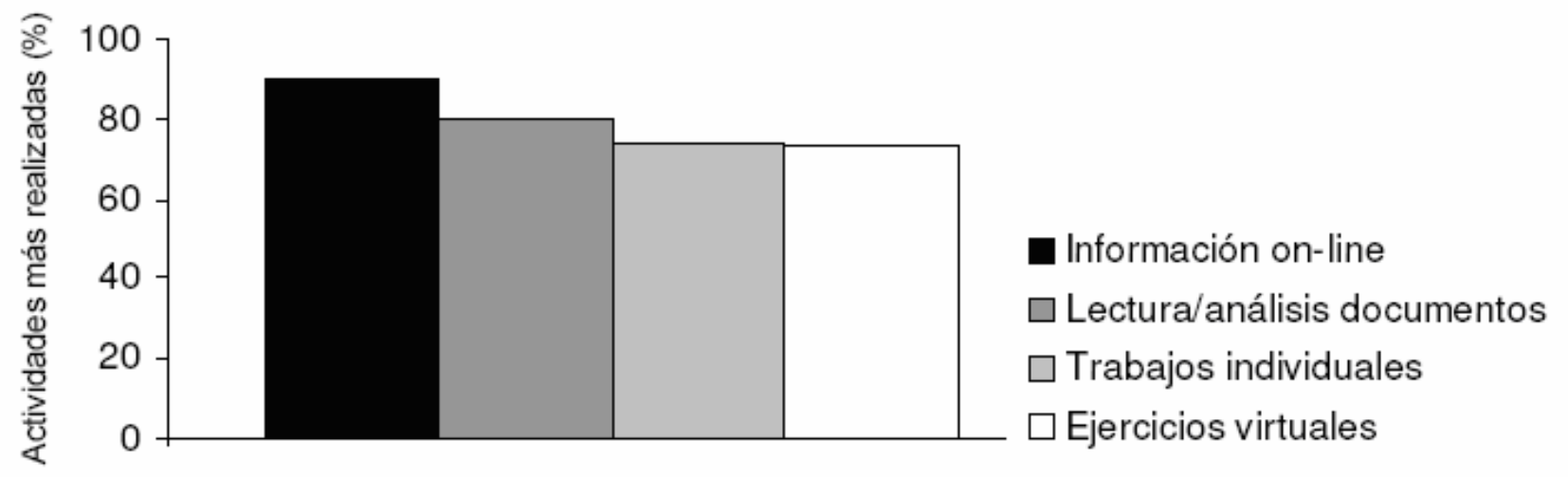

Fig. 1: Actividades más realizadas por el alumnado en las sesiones no presenciales

En nuestro quehacer diario, se contemplan como actividades no presenciales no sólo las derivadas de un trabajo online, aunque sin duda es la herramienta que más se usa, sobre todo mediante la utilización de la plataforma de teleformación Tem@, de la Universidad de Vigo. En ocasiones la sesión no presencial se estructura sin apoyo virtual y las actividades que se realizan se relacionan con el trabajo individual/grupal, el análisis y comprensión de documentos, la búsqueda de información, la realización de supuestos prácticos, etc. En la literatura existente, sin duda proliferan las investigaciones que utilizan la modalidad no presencial por medio de entornos virtuales sin recurrir a otras estrategias. Sin embargo, hay constancia de que estas intervenciones se dan, de manera que el sistema no presencial no queda reducido exclusivamente al uso de Internet o de plataformas virtuales de enseñanza-aprendizaje (Del Río, 2010).

Para lo que menos utilizan las sesiones no presenciales nuestros/as estudiantes es para buscar información en la biblioteca (54\%), realizar trabajos grupales (45\%), consultar dudas (30\%) y participar en foros/debates de la asignatura (8\%). Castañeda (2007) sostiene que la comunicación en línea entre estudiantes y profesorado se da más a través del correo electrónico y no tanto mediante otras herramientas como los foros. 
En nuestro caso, no se contemplan como actividades de primer orden los trabajos realizados en grupo y, como se verá más adelante, el trabajo en equipo es una de las competencias menos valoradas. Creemos que esta es una cuestión a tener en cuenta de cara al futuro, sobre todo si además se valoran experiencias como la de Alfageme (2005), realizada con universitarios de estudios impartidos en la Facultad de Educación de diferentes universidades españolas, en las que, aun diseñando experiencias educativas que plantean el trabajo de forma colaborativa, aportan resultados algo insatisfactorios respecto a cómo se trabaja en grupo y a la dificultad que supone que varias personas interaccionen juntas en línea.

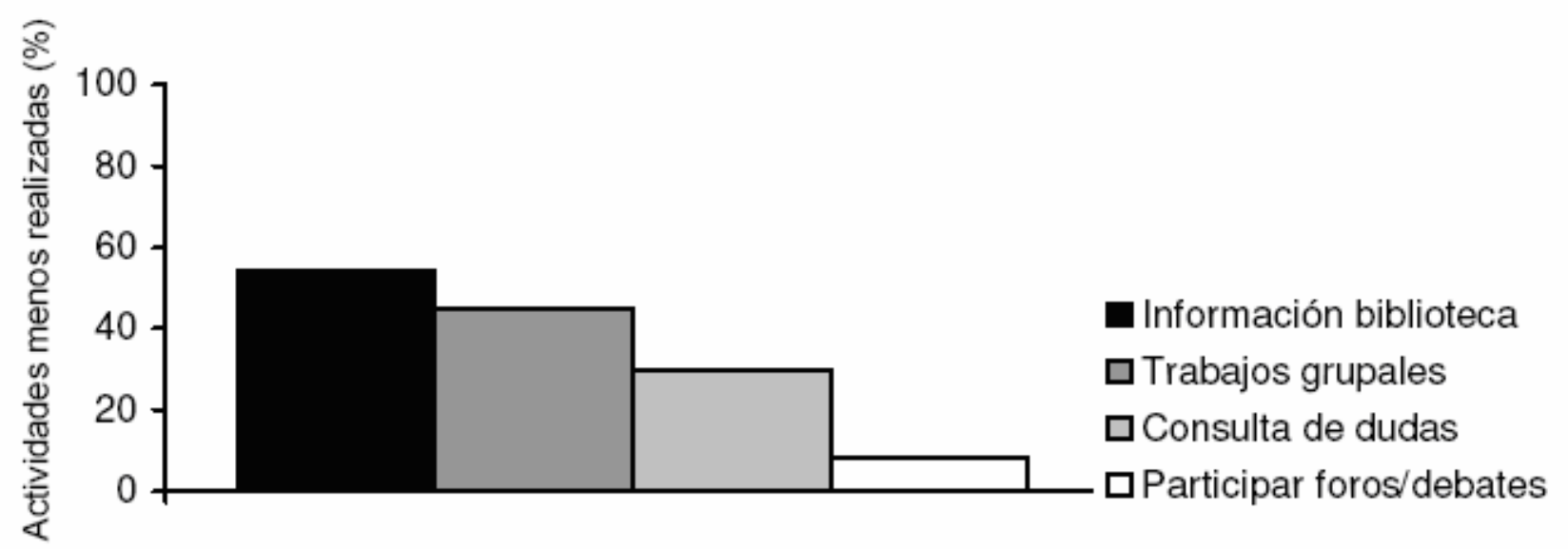

Fig. 2: Actividades menos realizadas por el alumnado en las sesiones no presenciales

Las tareas de esas clases no presenciales se realizaron mayoritariamente (64\%) en un horario diferente al de la asignatura, mientras que aproximadamente la cuarta parte de los estudiantes (27\%) utilizaba siempre ese horario en concreto para realizarlas. El 9\% llevaba a cabo las tareas tanto en las horas de la asignatura como en otras horas.

Otro tema interesante es el relativo a si estas clases contribuyen al desarrollo de ciertas competencias. Los ítems más valorados han sido los de aprendizaje autónomo (85\%), la búsqueda de información (81\%) y el uso de nuevas tecnologías (74\%). Estos hallazgos coinciden con los de Fernández-Villarino (2008), en un estudio realizado con estudiantes de cuarto curso de la licenciatura de Ciencias de la Actividad Física y del Deporte donde indica que los/as estudiantes valoran también en un alto grado competencias como el uso de las tecnologías de la información (TIC) y el aprendizaje autónomo (esta última también valorada por los/as estudiantes en García et al., 2008). Castañeda (2007), por su parte, comenta asimismo que el alumnado universitario es consciente de que las TIC son un recurso de trabajo y que dicho colectivo considera fundamental el hecho de poder trabajar con estas tecnologías.

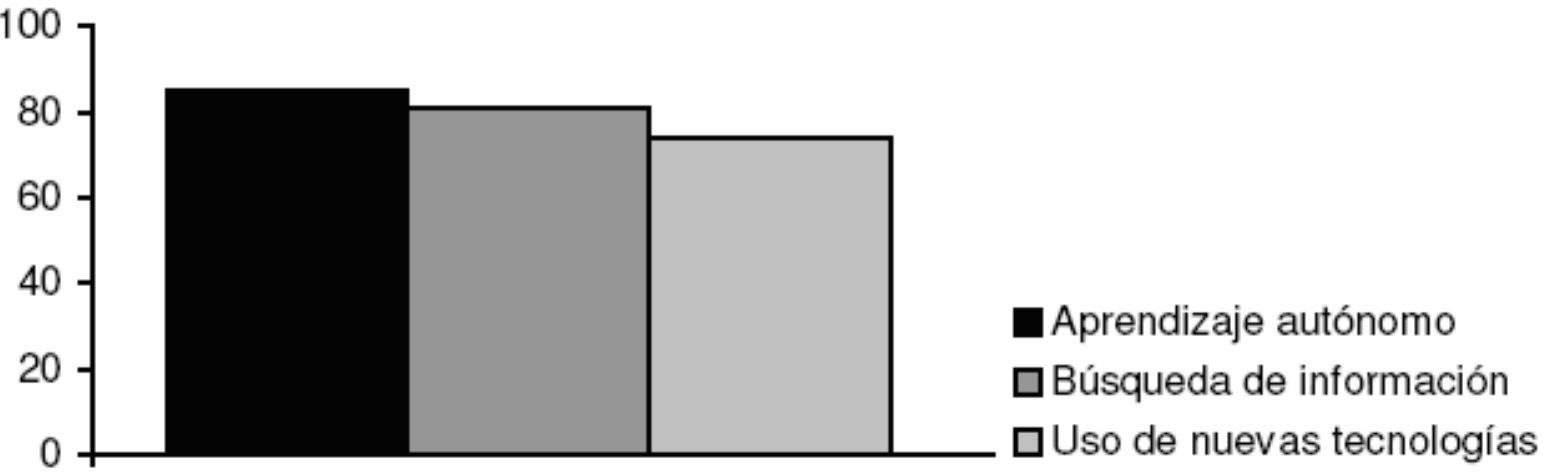

Fig. 3: Competencias más valoradas por el alumnado en el desarrollo de las sesiones no presenciales (\%) 
Los/as estudiantes perciben que durante el proceso llevado a cabo con este recurso se han desarrollado menos la reflexión-espíritu crítico (46\%), el trabajo en equipo (42\%) (ambas competencias valoradas de forma media) y muy poco las relaciones/comunicación entre el alumnado y entre el alumnado/profesorado, ya que estas últimas categorías han sido señaladas por apenas un $22-23 \%$ de las personas respectivamente.

Los datos, en general, no son coherentes con los aportados en la literatura revisada. Barceló et al. (2007) indican que las competencias más desarrolladas a través de las actividades formativas no presenciales son el pensamiento crítico y la gestión de la información; Fernández-Villarino (2008) observa como más valoradas, entre otras ya comentadas con anterioridad, las competencias que se vinculan al liderazgo, trabajo en equipo y relaciones interpersonales; y Blasco y Mengual (2007) apuntan que un $92 \%$ de los estudiantes consideran que los materiales de aprendizaje utilizados en su estudio permiten realizar una reflexión crítica de la propia práctica. Sin embargo, se tiene evidencia de trabajos que ofrecen resultados más en consonancia con los nuestros, como el de García et al. (2008) en el que las relaciones entre compañeros/as y docentes no son lo idóneas que se quisieran, detectándose problemas a la hora de unificar criterios, a la hora de coordinarse, de llegar a un consenso ante opiniones diversas, etc. Este aspecto ha sido poco valorado por las personas del estudio, al igual que el trabajo en equipo. Por tanto, es importante reflexionar sobre esta cuestión, pero a priori parece lógico pensar que la valoración de competencias va a depender directamente de las actividades programadas por el profesorado.

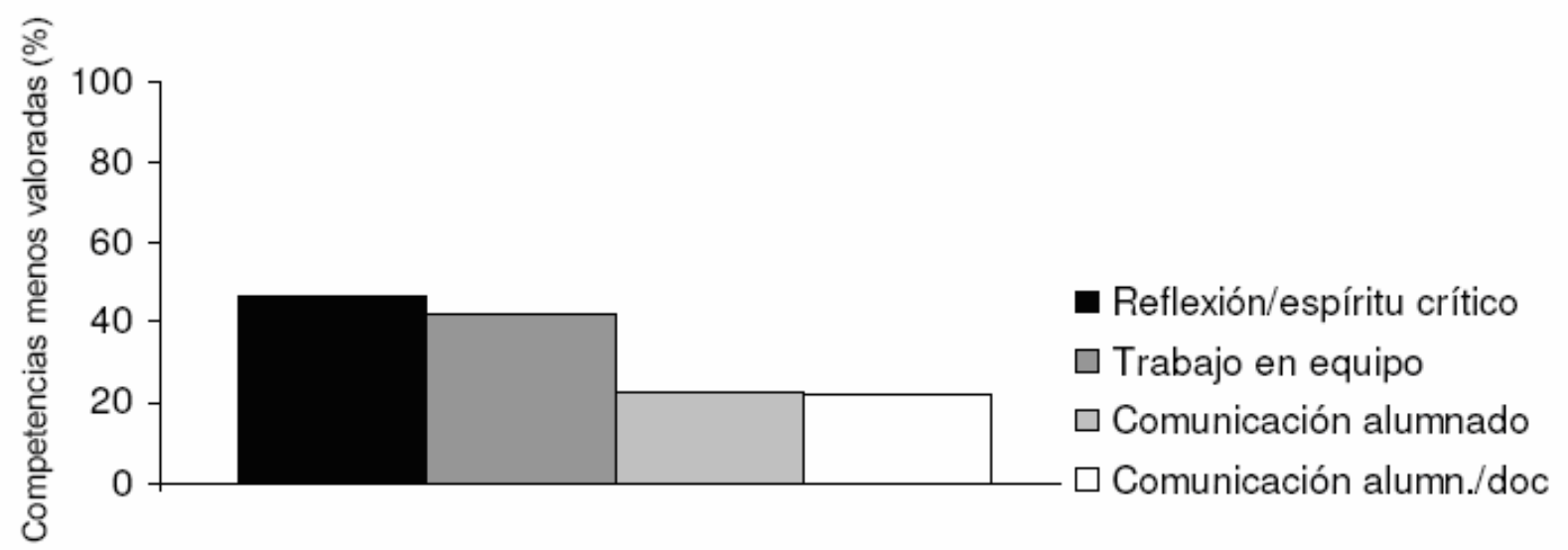

Fig. 4: Competencias menos valoradas por el alumnado en el desarrollo de las sesiones no presenciales (\%)

Una de las curiosidades que se planteaban era la de saber si para los estudiantes el hecho de hacer clases no presenciales suponía menos trabajo para el profesor; el $65 \%$ del alumnado consultado cree que no, sin embargo, el $26 \%$ opina que sí y un $9 \%$ no contesta a esta cuestión.

Cuando se les pregunta sobre si después de haber experimentado este tipo de sesiones consideran que ha mejorado su proceso de aprendizaje, el 90\% contesta que sí; además les gustaría disponer de más sesiones de este tipo a lo largo de su proceso formativo (80\%). Se encuentran los mismos resultados respecto a estas dos cuestiones y con porcentajes casi idénticos en Fernández-Villarino (2008) y de forma mayoritaria este tipo de modalidad se acoge como algo positivo y satisfactorio (Alados et al., 2010; Blasco y Mengual, 2007; Chiecher et al., 2005; Fernández-Villarino, 2008; Gras-Martí et al., 2004; Pérez-Lorido, 2008).

\section{CONCLUSIONES}

El alumnado considera que las sesiones no presenciales son una estrategia didáctica que contribuye a mejorar su proceso de enseñanza-aprendizaje.

El uso de las sesiones no presenciales favorece la adquisición de las competencias de autonomía y responsabilidad en el proceso de enseñanza-aprendizaje, uso de las nuevas tecnologías y capacidad para seleccionar la información adecuada. 
El tipo de actividades que fundamentalmente desarrollan los alumnos y las alumnas durante estas sesiones no presenciales son: lectura y/o análisis de documentos, búsqueda de información en Internet, realización y/o entrega de ejercicios mediante el uso de plataformas de teleformación.

Entendemos que el profesorado debe perfeccionar el planteamiento de las sesiones no presenciales en relación con: el trabajo en equipo, el uso de las tecnologías de la información y la comunicación, las relaciones entre estudiantes y los procesos de crítica y reflexión.

Sería deseable realizar una experiencia de carácter colaborativo por parte del profesorado, en donde de manera horizontal se planifiquen y se programen este tipo de sesiones.

\section{REFERENCIAS}

Agra, M.J., Gewerc, A. y Montero, M.L. El portafolios como herramienta de análisis en experiencias de formación on-line y presenciales. Enseñanza \& Teaching, 21, 2003. http://fineans.usac.edu.gt/elgg/comunidad/files/ 2084/9634/portafolio.pdf. Acceso: 14 de febrero de 2011.

Alados, I., Liger, E. y Peula, J.M. Uso de herramientas del Campus Virtual de la Universidad de Málaga (UMA) en la docencia de asignaturas de física. En La innovación educativa en el contexto actual de la educación superior, pp. 643-646. Vigo, España (2010).

Alfageme, M.B. El trabajo colaborativo en situaciones no presenciales. Pixel-Bit: Revista de medios y educación, (en línea), 26, 2005. http://www.sav.us.es/pixelbit/pixelbit/ articulos/n26/n26art/art2601.htm. Acceso: 16 de febrero de 2011.

Barceló, O., Olivan, J., Asensio, A. y González-Castañón, J.A. Actividades formativas no presenciales. Una experiencia de la Facultad de Ciencias de la Actividad Física y del Deporte en la Universidad Europea de Madrid. Lecturas: Educación física y deportes, (en línea), 105, 2007. http://dialnet.unirioja.es/servlet/articulo?codigo=2236556. Acceso: 17 de marzo (2009).

Blasco, J.E. y Mengual, S. La observación sistemática como medio de adquisición del conocimiento en las sesiones presenciales y no presenciales de los alumnos en la asignatura de Educación Física y su Didáctica II. (2007). http://www.eduonline.ua.es/jornadas2007I comunicaciones/2B6.pdf. Acceso: 13 de febrero de 2011.

Bolívar, A. La planificación por competencias en la reforma de Bolonia de la Educación Superior. Un análisis crítico. En Diseñar y evaluar por competencias en la Universidad. El EEES como reto, pp. 13-18. Vigo, España (2009).

Castañeda, L.J. Implementación de materiales en red en contextos universitarios presenciales. La perspectiva del alumnado. Elearning Papers, (en línea), 4, 2007. http://www.elearningeuropa.info/files/media/media12743.pdf. Acceso: 16 de febrero de 2011.

Chiecher, A., Donolo, D. y Rinaudo, M.C. Percepciones del aprendizaje en contextos presenciales y virtuales. La perspectiva de alumnos universitarios. RED, Revista de Educación a Distancia, (en línea), 13, 2005. http://www.um.es/ead/red/13/chiecher.pdf. Acceso: 17 de marzo (2009).

Cohen, L. y Manion, L., Métodos de investigación educativa. La Muralla, S.A., Madrid, España (1990).

De Miguel, M. Modalidades de enseñanza centradas en el desarrollo de competencias. Orientaciones para promover el cambio metodológico en el Espacio Europeo de Educación Superior (2005), Proyecto EA2005-0118. (2005). http://www.ub.edu/oce/documents/pdfes/mec/ mec_2005_comp.pdf. Acceso: 17 de marzo (2009). 
DB, Declaración de Bolonia. El Espacio Europeo de la Enseñanza Superior. (1999).

http://www.crue.org/export/sites/Crue/procbolonia/documentos/docclave/Declaracixn_de_Bolonia.p df Acceso: 9 de octubre de 2009.

Del Río, P. Elaboración de material didáctico: como enseñar el teatro de sombras, una experiencia con alumnos de Ciencias de la Educación. En La innovación educativa en el contexto actual de la educación superior, pp. 229-231. Vigo, España (2010).

Fernández March, A. A profesionalización da docencia: a resposta aos retos do proceso de converxencia europea. Colección Formación e Innovación Educativa na Universidade, Universidade de Vigo, Vigo, España (2008).

Fernández March, A. Nuevas metodologías docentes (2006), http://www.upm.es/innovacion/cd/ 02_formacion/talleresnuevas_meto_docent/nuevas_metodologias_docentes_2.pdf. Acceso: 17 de marzo (2009).

Fernández-Villarino, M.A. As actividades non presenciais. Un pequeño paso nas estrategias didácticas do Espazo Europeo de Educación Superior. En Prácticas Educativas Innovadoras na Universidade, pp. 245-259. Vigo, España (2008).

Gallego, M.J. Las funciones docentes presenciales y virtuales del profesorado universitario. Revista electrónica Teoría de la Educación. Monográfico: Educación y Cultura en la Sociedad de la Información, (en línea), 8(2), 2007. http://campus.usal.es/ -teoriaeducacion/ rev_numero_08_02/n8_02_arrufat.pdf. Acceso: 20 de marzo (2009).

García, C., Oltra, M.J., Flor, M.L. y Boronat, M. Autoaprendizaje y desarrollo de competencias en grupos de trabajo presenciales y no presenciales. Jornadas de Investigación en Docencia Universitaria : la construcción colegiada del modelo docente universitario del siglo XXI. (2008). http://www.eduonline.ua.es/jornadas2008/comunicaciones/3E2.pdf. Acceso: 20 de marzo (2009).

Gras-Martí, A., Cano-Villalba, M. y Cano, C. Cursos de TIC per al professorat: anàlisi comparatiu de les modalitats presencial, semipresencial i no presencial. Revista Electrónica de Enseñanza de las Ciencias, (en línea), 1, 2004. http://reec.uvigo.es/volumenes/volumen3/Numero1/Art3.pdf. Acceso: 20 de marzo (2009).

Lamas, M.L., Massié, A.I. y Quero, E.D. Implementación de un aula virtual bajo la modalidad mixta: El Caso de Química Agrícola en la Universidad Nacional de Salta. Formación Universitaria, (en línea), 3(4), 2010. http://www.citrevistas.cl/revista-formacion/v3n4fu/art02.pdf. Acceso: 2 de marzo (2011)

Lovaina, Comunicado de Lovaina: El Proceso de Bolonia 2020. El Espacio Europeo de Educación Superior en la nueva década. (2009).

Mondéjar, J.A., Mondéjar, J. y Vargas, M. Docencia virtual en universidades presenciales: experiencia en la Universidad de Castilla-La Mancha. RIED: Revista Iberoamericana de Educación a Distancia, (en línea), 10(2), 2007.

http://www.utpl.edu.ec/ried/images/pdfs/volumendiez/docencia-virtual.pdf. Acceso: 2 de enero de 2011.

Pérez-Lorido, M. Asignaturas virtuales en universidades presenciales: perspectivas y problemas. Pixel-Bit: Revista de medios y educación, (en línea), 30, 2007.

http://www.sav.us.es/pixelbit/pixelbit/articulos/n31/n31art/art3115.htm. Acceso: 1 de marzo de 2011.

Pérez-Lorido, M. Campus virtuales en universidades presenciales: ¿sueñan los estudiantes con profesores eléctricos? RELATEC: Revista Latinoamericana de Tecnología Educativa, 7(1), 2008. 
Sangrà, A. Educación a distancia, educación presencial y uso de la tecnología: una tríada para el progreso educativo. Seminario: La utilización de las tecnologías de la información y la comunicación en Educación Superior: un enfoque crítico, Univ. Autónoma de Madrid Mayo (2002).

Santángelo, H.N., Modelos pedagógicos en los sistemas de enseñanza no presencial basados en nuevas tecnologías y redes de comunicación. Revista Ibero-Americana de Educación, (en línea), 24, 2000. http://www.rieoei.org/rie24a06.PDF. Acceso: 20 de marzo (2009).

Zabalza, M.A., Competencias docentes del profesorado universitario: calidad y desarrollo profesional. Narcea, Madrid, España (2003). 